\title{
Long-term Stability of Ectasia in a Young Patient with Asymmetric Keratoconus
}

\author{
${ }^{1}$ Rosane de Oliveira Corrêa, ${ }^{2}$ Ana Laura Caiado Canedo, ${ }^{3}$ Rozalia Beildeck, ${ }^{4}$ Marcella Quaresma Salomão \\ ${ }^{5}$ Penelope Burle de Politis, ${ }^{6}$ Renato Ambrósio Jr
}

\begin{abstract}
Purpose: To report the clinical course of a typical young patient presenting with asymmetric keratoconus (KC), that demonstrates that stabilization of the ectatic process is possible without cross-linking (CXL) procedure.
\end{abstract}

Methods: Case report and review of the literature.

Results: A 17-year-old male patient was referred due to the diagnosis of keratoconus. Patient complained of loss of vision in the left eye (OS). Uncorrected distance visual acuity (UDVA) was $20 / 25+$ in the right eye (OD) and 20/80 in the left eye; wavefront-assisted manifest refraction gave best corrected distance visual acuity (CDVA) of 20/20 in OD and 20/40 in OS. The diagnosis of keratoconus was confirmed with Placido disk-based topography (Oculus Keratograph 4), and Pentacam HR corneal tomography (Oculus Optikgeräte $\mathrm{GmbH}$, Wetzlar, Germany). Femtosecond laser-assisted intracorneal ring segment (ICRS) implantation was performed in the left eye and treatment for allergy was prescribed for both eyes, along with patient education and advice not to rub the eyes. After 3 months, significant improvement was observed on UDVA $(20 / 30)$ and CDVA (20/20) in the left eye. Topometric and tomographic stability of ectasia was observed in the right eye in a 4-year follow-up.

Conclusion: Intracorneal ring segment caused significant regularization of the corneal shape and improvement on visual acuity. Ectasia stability was achieved with no need for CXL, despite the patient's young age. This case raises the point that the indication of CXL for every keratoconic patient should be reconsidered.

Keywords: Cross-linking indication, Intracorneal ring, Keratoconus.

How to cite this article: de Oliveira Corrêa R, Canedo ALC, Beildeck R, Salomão $M Q$, de Politis $\mathrm{PB}$, Ambrósio R Jr. Longterm Stability of Ectasia in a Young Patient with Asymmetric Keratoconus. Int J Kerat Ect Cor Dis 2015;4(2):66-68.

\footnotetext{
${ }^{1-5}$ Research Associate, ${ }^{6}$ Founder and Director, Associate Professor

${ }^{1-5}$ Corneal Tomography and Biomechanics Study Group, Rio de Janeiro, Brazil

${ }^{6}$ Corneal Tomography and Biomechanics Study Group, Rio de Janeiro, Federal University of São Paulo, São Paulo, Brazil

Corresponding Author: Renato Ambrósio Jr, PhD, Instituto de Olhos Renato Ambrósio, Rua Conde de Bonfim 211/712-20520-050, Phone/Fax: 55212234-4233, e-mail: dr.renatoambrosio@gmail.com
}

\section{Source of support: Nil}

Conflict of interest: Dr Ambrósio is a consulant for oculus and wavelight-alcon

\section{INTRODUCTION}

Keratoconus $(\mathrm{KC})$ is a corneal ectatic disorder that some regard as a new subspeciality in ophthalmology. It usually presents bilaterally with asymmetry that is characterized by the degree of chronic biomechanical failure that has occurred in each eye. It generally progresses to corneal thinning and forward protusion of the cornea, culminating in loss of visual acuity due to irregular astigmatism and myopia. ${ }^{1}$ Advances in diagnostic methods for detecting the corneal ectatic processes using enhanced refractive surgery screenings tools allows surgeons the ability to detect both the disease earlier than previously found, often times in the stage of KC susceptibility. ${ }^{2}$ The treatment of $\mathrm{KC}$ has been changing and is based on the stage of the ectatic condition. ${ }^{3}$ In the disease onset, glasses and/or contact lenses are the first line treatment to correcting astigmatism caused by the ectatic process satisfactorily. When contact lenses are no longer effective to provide enough best corrected distance visual acuity or in cases of intolerance to contact lenses, intracorneal ring segment (ICRS), implantation is recommended. In the past, there was no treatment to improve the chronic corneal biomechanical failure process or even to stop the disease progression. Thus, corneal transplantation was considered the conventional surgical therapeutical approach for the disorder. ${ }^{3}$ Corneal collagen cross-linking (CXL) emerged as a novel treatment for $\mathrm{KC}$ since it is able to stop KC progression by increasing corneal biomechanical stiffness and strength; it has also been recommended by some experts for young patients to halt the ectatic process. $^{4-8}$

\section{CASE REPORT}

A 17-year-old male patient was referred for ophthalmological evaluation due to the diagnosis of $\mathrm{KC}$ and reduced vision in the left eye in 2010. Complete eye exam was performed and the diagnosis of KC was confirmed using the Placido disk-based topography (Oculus Keratograph 5) and Pentacam HR corneal tomography (Oculus Optikgeräte GmbH, Wetzlar, Germany; Fig. 1). 


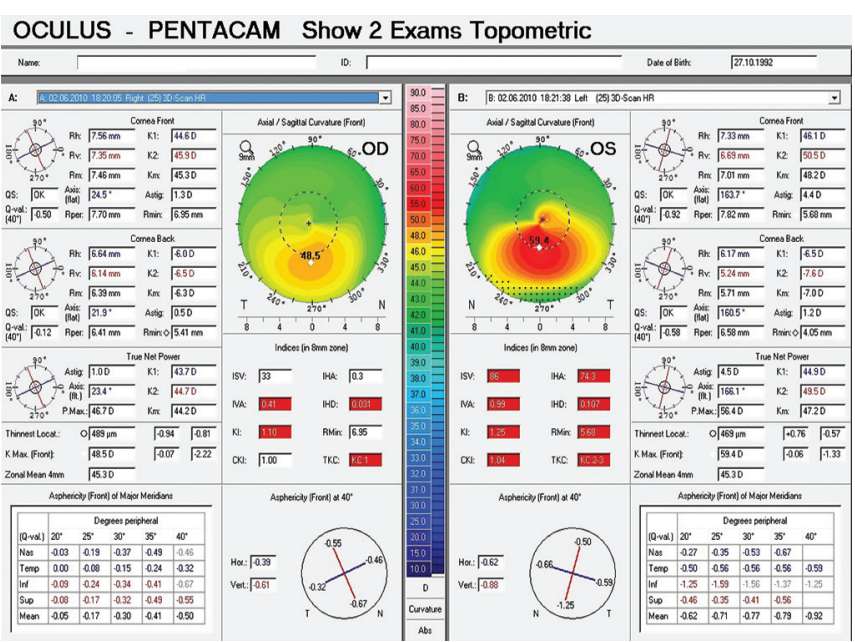

Fig. 1: Oculus Pentacam sagittal curvature maps of $\mathrm{OU}$ in asymmetric keratoconus before treatment. UCVA was 20/20 OD and $20 / 80$ OS

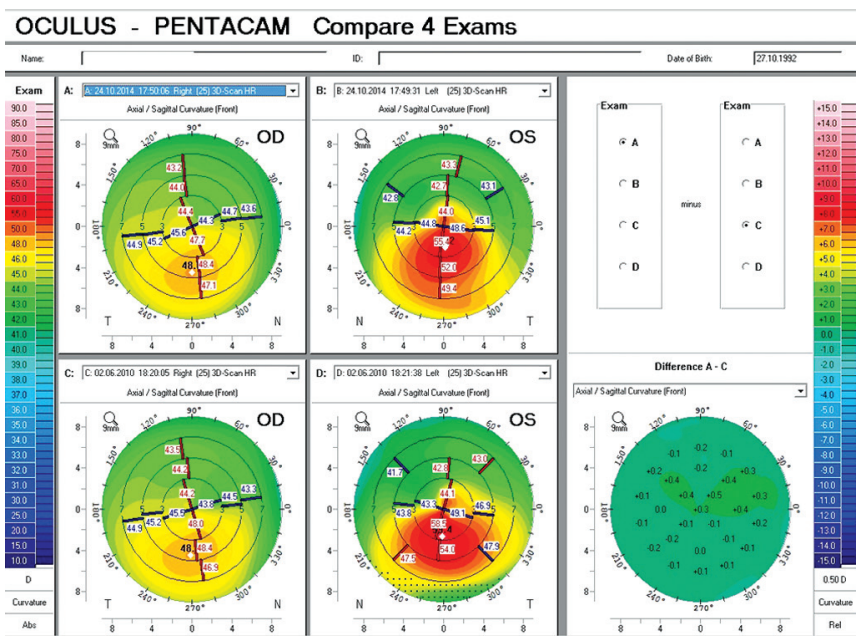

Fig. 3: OU Oculus Pentacam sagittal curvature maps in asymmetric keratoconus. Preoperatively (bottom): OD and OS (C and D respectively). After 4 years of follow-up (top): OD and OS (A and B, respectively). Difference A-C map shows the corneal stability OD

Uncorrected distance vision acuity (UDVA) was 20/20 OD and 20/80 OS; wavefront-assisted manifest refraction provided corrected distance visual acuity (CDVA) of $20 / 20(-0.25$ sph -0.25 cyl 52) in the right eye and 20/40 $(+0.25 \mathrm{sph}-2.75 \mathrm{cyl} 165)$ in the left eye.

Indication for surgery in OS was based on the unsatisfactory visual acuity with glasses and the contact lens intolerance. Femtosecond laser-assisted intrastromal corneal ring segment (ICRS) implantation was performed in the left eye. The iFS $150 \mathrm{kHz}$ (Abbot Medical Optics, Santa Ana, CA) was used to create a $360^{\circ}$ tunnel at $75 \%$ depth of the minimal pachymetry on the Pentacam, with incision at the steep axis for implanting one segment of Keraring SI $150^{\circ}$ with $250 \mu \mathrm{m}$ (Mediphacos, Belo Horizonte, Brazil).

Topical treatment for chronic allergy with multifunctional agents and corneal ocular surface optimization with omega-3 essential fatty acid oral supplementation were prescribed. The patient was stoutly advised not to

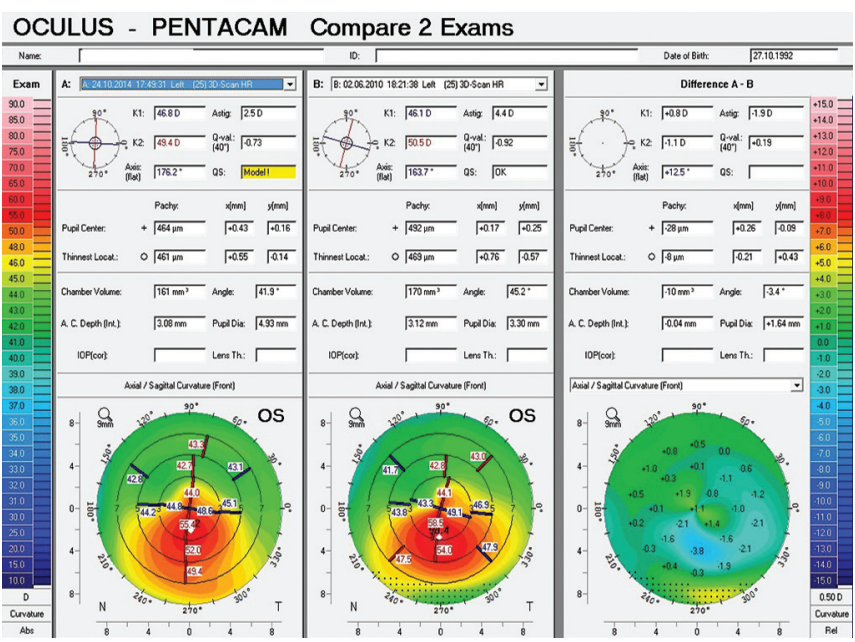

Fig. 2: OS Oculus Pentacam sagittal curvature maps in asymmetric keratoconus preoperatively (B) and 4 years after ICRS segment implantation (A). Difference A-B map shows the flattening effect

rub the eyes. Clinical follow-up was suggested for the fellow right eye which had mild KC and still retained satisfactory uncorrected vision.

After ICRS implantation in OS, UDVA improved to 20/30 and remained stable from the third month as was the CDVA which improved to $20 / 20(+0.50 \mathrm{sph}-1.0$ cyl 128). The patient has been followed for over 4 years (52 months) with periodical exams, including corneal topography and tomography every 6 months. OD remained relatively stable (Figs 1 to 3; and Graphs 1 and 2), retaining UDVA 20/20.

\section{DISCUSSION}

Keratoconus is the most common corneal dystrophy. ${ }^{1}$ The hallmark of the disease is the chronic biomechanical failure process resulting in progressive thinning and anterior corneal protrusion, causing irregular astigmatism. ${ }^{1}$ Patients with signs of KC generally present in their twenties, associated with signs of allergic process, history of eye rubbing, and complaints of progressive blurred vision secondary to myopia and irregular astigmatism.,10 Treatment for ectatic disorders is based on correcting both their irregular astigmatism and corneal biomechanical instability. ${ }^{3,11}$ Patient education aimed for warning the patient not to rub the eyes and the allergy control are fundamental. ${ }^{9,10}$

Intrastromal corneal ring segment, implantation, regarded as an effective, safe and reversible surgical technique, was performed in the left eye to reduce corneal irregularity and, thus, avoiding corneal transplantation. ${ }^{12}$ Collagen cross-linking $(\mathrm{CX})$ is a safe and effective technique for halting the progression of corneal ectatic disorders by improving cornea biomechanical properties. ${ }^{4-8}$ Indication for CXL is considered to reduce 


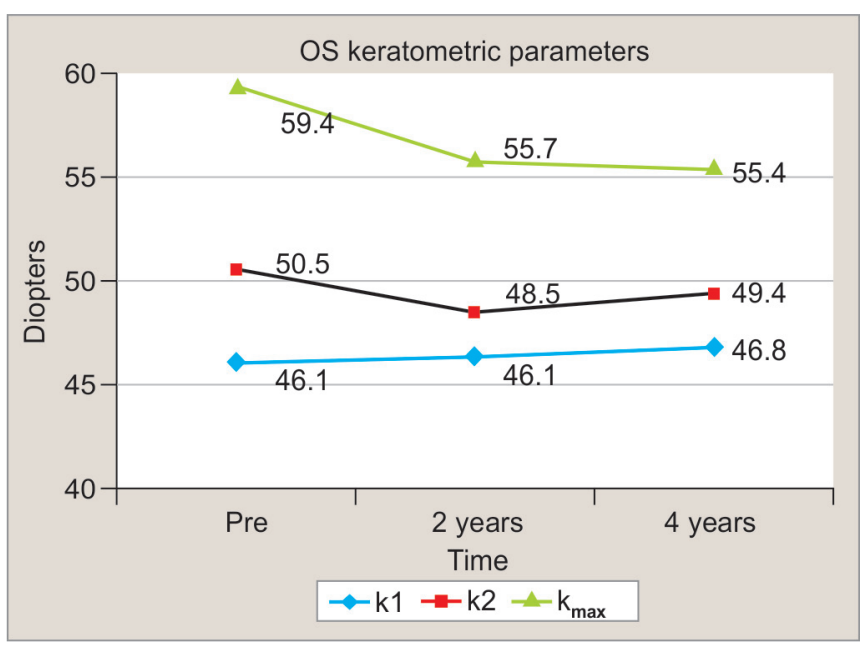

Graph 1: OS keratometric readings: preoperatively, 2 and 4 years after ICRS segment implantation

ectasia progression, which was not observed in this case after treatment.

There is a recent worldwide trend and experts have been discussing the recommendation for the procedure in progressive or non-progressive $\mathrm{KC}$ patients under the age of 21 to 25 since the disease is more prevalent and shows a higher risk of progressing in this group of individuals. ${ }^{13,14}$ Interestingly, Koller et al in 2009 evaluated the complication rate of CXL (percentage of eyes losing 2 or more Snellen lines) in 117 eyes (99 patients) with primary keratectasia and concluded that patients older than 35 years and with preoperative DCVA better than 20/25 were identified as being high risk for complications, and maximum keratometry reading more than 58.0 diopters was related to a significant risk for continued ectasia progression. ${ }^{15}$ Patients must be aware of risks and benefits and surgeons have to balance the real need to perform CXL treatment, especially if the patient has better than 20/25 visual acuity.

In this case report, ICRS implantation resulted in safe and effective regularization of the corneal shape, allowing improvement on both UCVA and DCVA OS. Ectasia stability was achieved with no need for CXL despite young age in the right eye over long-term followup. Maintenance of UCVA of 20/20 and no changes on tomographic maps after clinical treatment for allergy in 4-year follow-up has shown no need for CXL so far.

This case emphasizes that the indication of CXL for every KC patient should be reconsidered. Patients and parents deserve to have proper educational about the disease course, risk of progression and possible alternatives. These measures, along with allergy control, enabling the patient to reduce eye rubbing are extremely important for stabilization of ectasia. Of course, clinical followup with complementary examination, such as corneal topography and tomography needs to be continued.

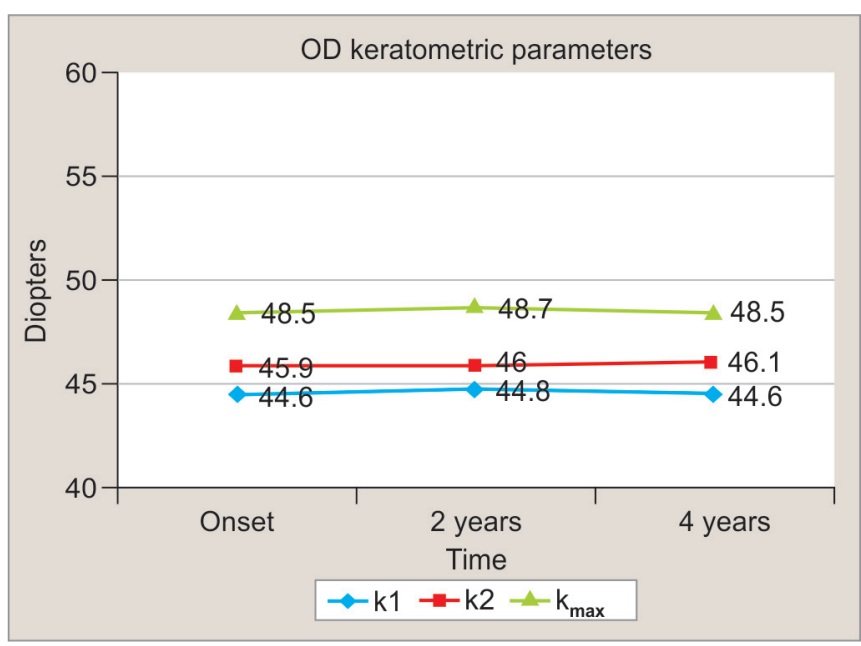

Graph 2: OD keratometric readings: onset, 2 and 4 years after ocular allergy control

\section{REFERENCES}

1. Rabinowitz YS. Keratoconus. Surv Ophthalmol 1998 Jan-Feb; 42(4):297-319.

2. Ambrosio R Jr. Simplying ectasia screening with Pentacam corneal tomography. Highlights of Ophthalmology 2010; 38(3):12-20.

3. Jhanji V, Sharma N, Vajpayee RB. Management of keratoconus: current scenario. Br J Ophthalmol 2011;95(8):1044-1050.

4. Spoerl E, Huhle M, Seiler T. Induction of cross-links in corneal tissue. Exp Eye Res 1998 Jan;66(1):97-103.

5. Spoerl E, Seiler T. Techniques for stiffening the cornea. J Refract Surg 1999;15:711-713.

6. Wollensak G, Spoerl E, Seiler T. Stress-strain measurements of human and porcine cornea after riboflavin/ultraviolet-Ainduced cross-linking. J Refract Surg 2003;29:1780-1785.

7. Wollensak G, Spoerl E, Seiler T. Riboflavin/ultravioletA-induced collagen cross-linking for the treatment of keratoconus. Am J Ophthalmol 2003;135:620-627.

8. Caporossi A, Baiocchi S, Mazzotta C, et al. Parasurgical therapy for keratoconus by riboflavin-ultraviolet type A rays induced cross-linking of corneal collagen. J Cataract Refract Surg 2006;32:837-845.

9. Bawazeer AM, Hodge WG, Lorimer B. Atopy and Keratoconus: a multivariate analysis. Br J Ophthalmol 2000;84:834-836.

10. Krachmer JH. Eye rubbing can cause keratoconus. Cornea 2004;23:539-540.

11. Wollensak G, Iomdina E. Biomechanical and histological changes after corneal cross-linking with and without epithelial debridement. J Cataract Refract Surg 2009;35: 540-546.

12. Kymionis GD, Siganos CS, Tsiklis NS, et al. Long-term followup of intacs in keratoconus. Am J Ophthalmol 2007 Feb;143(2): 236-244.

13. Vinciguerra P, Alb'e E, Frueh BE, Trazza S, Epstein D. Two-year corneal cross-linking results in patients younger than 18 years with documented progressive keratoconus. Am J Ophthalmol 2012 Sep;154(3):520-526

14. Gaster RN, Rabinowitz YS, Canedo AL. Corneal cross-linking in teenagers. Presented at the cross-linking congress, Deer Valley, Utah, 2012 July 29.

15. Koller T, Mrochen M, Seiler T. Complication and failure rates after corneal cross-linking. J Cataract Refract Surg 2009 Aug;35(8):1358-1362. 